\title{
Accretion and pulsation of IU Per based on INTEGRAL and ground base photometry
}

\author{
Emil Kundra ${ }^{1}$, Ladislav Hric ${ }^{1}$ \& Rudolf Gális ${ }^{2} \dagger$ \\ ${ }^{1}$ Astronomical Institute of the Slovak Academy of Sciences, 05960 Tatranská Lomnica, \\ Slovakia email: kundra@ta3.sk \\ ${ }^{2}$ Department of Theoretical Physics and Astrophysics, Institute of Physics, \\ Faculty of Sciences, P. J. Šafárik University, Park Angelinum 9, 04001 Košice, Slovakia
}

\begin{abstract}
IU Persei (IU Per) is an eclipsing semi detached (SD) binary with pulsating component (oscillating eclipsing Algol - oEA). Using our ground base photometry in Johnson's $B$ and $V$ filters and INTEGRAL/OMC (Optical Monitoring Camera) data in $V$ filter we introduce the model of this system. We determined the $(\mathrm{O}-\mathrm{C})$ diagram behavior. Moreover, the short term variations of the light curve were detected. Whats more, we obtained residua, by comparison of observational and synthetic light curves (LC), of which the detailed period analysis enabled to characterize and localize their source, that can be explained as the pulsation activity of the primary component. The secondary, less massive component, is beyond the instability belt on H-R diagram and fulfills its Roche lobe. The accreting primary component pulsates in modes typical for the $\delta$ Scuti stars.
\end{abstract}

Keywords. binaries: eclipsing stars: oscillations, accretion, techniques: photometric

\section{Introduction, data and analysis}

IU Per is an oscillating eclipsing Algol - oEA, of spectral type A4+G6IV. The orbital period of $0.8570257 \mathrm{~d}$ and HJD 2452500.214 was determined by Kreiner (2004). Qian (2001) determined the change of period and calculated the quadratic ephemeris. Previous results were used by Kim et al. (2005), who analyzed the photometric observations of IU Per and detected two frequencies $f_{1}=42.103 \mathrm{c} / d$ (cycles per day) and $f_{2}=45.806 \mathrm{c} / d$. They classified the primary of IU Per, on which the mass accretion happens in SD system, as the pulsating variables of $\delta$ Scuti. Zhang et al. (2009) used 16 new published minima and added 7 new and calculated the new linear and quadratic ephemeris. They determined the parameters of the system and approved that the system was SD and the secondary component fulfilled its Roche lobe. The secondary is beyond the instability belt on $\mathrm{H}$ - $\mathrm{R}$ diagram and therefore the pulsating and mass accretion star is the primary.

We obtained the data at the Observatories of Astronomical Institute of the Slovak Academy of Sciences which cover its 7 primary minima and from INTEGRAL satellite. To observe the changes of $(\mathrm{O}-\mathrm{C})$ diagram of IU Per in the longest time interval we used all possible data published in the literature (173 times of primary and 7 times of secondary minima). We constructed the (O - C) diagram according to the linear ephemeris Kreiner (2004) which is sufficient for the LC modelling and analysis of the residua. The variations of residua vanished around the primary minima in our data, therefore the part of the LC was removed from the data. We detected periods in interval $0.3-0.65 \mathrm{~h}$ with

$\dagger$ This article was supported by the VEGA Grant No.2/0078/10 and by the realization of the Project ITMS No. 26220120029, based on the supporting operational Research and development program financed from the European Regional Development Fund. 


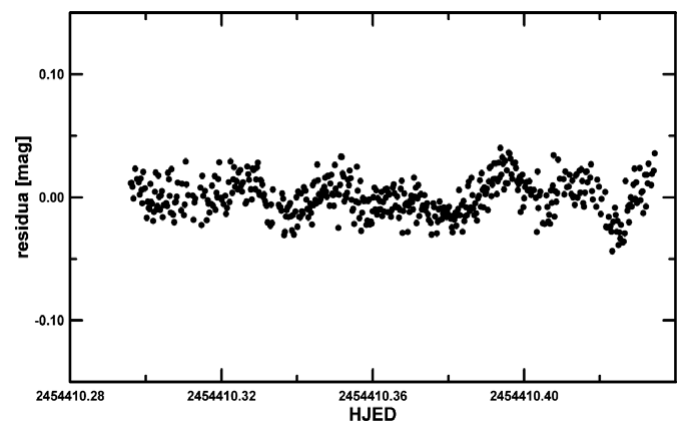

Figure 1. The residua show the pulsation activity of IU Per observed on 5. November 2007.

two significant frequencies in each data set. These periods corresponds to the pulsations of the $\delta$ Scuti stars, therefore the primary is probably the same type. Periods of pulsations obtained in this paper do not contradict the results recently published by Kim et al. (2005) and Zhang et al. (2009). We identified the following modes of pulsations: the fifth harmonic radial mode $5 H$ and the seventh harmonic non-radial mode of pulsations $p 7$. The primary star is lying on the left from the main sequence of the stars of the $\delta$ Scuti what was in coincidence with our results of period analysis where the primary of IU Per was not pulsating in the base mode, but in higher harmonic.

We got 49 photometric data obtained from August 1st till August 4th 2004 from the OMC archive. The data distribution does not allow to determine the exact times of minima, but the synthetic LC is in coincidence with ground base observations. The data in positions out of primary eclipses have higher scatter what is probably caused by pulsation activity of the primary component of the system. The period analysis of the LCs residua gave us the best periods about 0.075 and $0.061 \mathrm{~d}$. We do not consider the obtained periods to be real in comparisson with results obtained by period analysis of ground based photometric observations. These periods very probably relate with data distribution from OMC.

\section{Conclusions}

In the case of interacting eclipsing binary IU Per we were aimed to obtain the LC in Johnson's $B$ and $V$ and to construct the synthetic LC. We obtained the residua, which reflected the short term photometric variations and study the physical mechanism, which caused them. The analysis gave us the period of variations in the interval from 0.30 to 0.65 h. There were mostly two significant periods in the selected nights, while in individual nights their values were slightly shifted. With respect to vanishing of these variations during the primary minima we deduced that they were localized on the primary. The position of the primary in H-R diagram is on the left from main sequence of $\delta$ Scuti stars what is in coincidence with its pulsations in higher harmonic modes, where one mode is radial and the second is non-radial. In the future it is chance to study the long term changes caused by interaction of the IU Per binary.

\section{References}

Kim, S. L., Lee, J. W., Koo, J. R., Kang, Y. B., \& Mkrtichian, D. E. 2005, IBVS, 5629

Kreiner, J. M. 2004, Acta Astronomica, 54, 207

Qian, S. 2001, Astronomical journal, 121, 1614

Zhang, X. B., Zhang, R. X., \& Li, Q. S. 2009, Research in Astronomy \& Astrophysics, 9, 422 